\title{
Frustrated Heisenberg antiferromagnet on the honeycomb lattice: A candidate for deconfined quantum criticality
}

\author{
D. J. J. Farnell, ${ }^{1}$ R. F. Bishop, ${ }^{2}$ P. H. Y. Li,${ }^{2}$ J. Richter, ${ }^{3}$ and C. E. Campbell ${ }^{4}$ \\ ${ }^{1}$ Division of Mathematics, University of Glamorgan, Pontypridd CF37 1DL, Wales, UK \\ ${ }^{2}$ School of Physics and Astronomy, The University of Manchester, Manchester M13 9PL, UK \\ ${ }^{3}$ Institut für Theoretische Physik, Otto-von-Guericke Universität Magdeburg, P.O.B. 4120, D-39016 Magdeburg, Germany \\ ${ }^{4}$ School of Physics and Astronomy, University of Minnesota, Minneapolis, Minnesota 55455, USA \\ (Received 20 March 2011; revised manuscript received 20 May 2011; published 8 July 2011)
}

\begin{abstract}
We study the ground-state (gs) phase diagram of the frustrated spin- $\frac{1}{2} J_{1}-J_{2}-J_{3}$ antiferromagnet with $J_{2}=$ $J_{3}=\kappa J_{1}$ on the honeycomb lattice using coupled-cluster theory and exact diagonalization methods. We present results for the gs energy, magnetic order parameter, spin-spin correlation function, and plaquette valence-bond crystal (PVBC) susceptibility. We find a Néel antiferromagnetic (AFM) phase for $\kappa<\kappa_{c_{1}} \approx 0.47$, a collinear striped AFM phase for $\kappa>\kappa_{c_{2}} \approx 0.60$, and a paramagnetic PVBC phase for $\kappa_{c_{1}} \lesssim \kappa \lesssim \kappa_{c_{2}}$. The transition at $\kappa_{c_{2}}$ appears to be of first-order type, while that at $\kappa_{c_{1}}$ is continuous. Since the Néel and PVBC phases break different symmetries our results favor the deconfinement scenario for the transition at $\kappa_{c_{1}}$.
\end{abstract}

DOI: 10.1103/PhysRevB.84.012403

PACS number(s): 75.10.Jm, 03.65.Ca, 75.50.Ee

Frustrated quantum Heisenberg magnets are paradigms of systems that may be used to study quantum phase transitions (QPTs) between quasiclassical magnetically ordered phases and magnetically disordered quantum phases. In particular, if the quasiclassical phase and the quantum phase spontaneously break different symmetries, the Landau-Ginzburg-Wilson scenario of continuous phase transitions does not hold and the concept of deconfined quantum criticality ${ }^{1}$ becomes relevant. A canonical model for studying such QPTs is the spin- $\frac{1}{2}$ Heisenberg antiferromagnet (HAFM) with antiferromagnetic (AFM) nearest-neighbor (nn) $J_{1}$ coupling and frustrating AFM next-nearest-neighbor (nnn) $J_{2}$ coupling (namely the $J_{1}-J_{2}$ model) on the square lattice (see, e.g., Refs. 2-7). This model undergoes a transition from a quasiclassical Néel state at $J_{2} / J_{1} \lesssim 0.4$ to a quantum paramagnetic (QP) phase, which is not magnetically ordered, for $0.4 \lesssim J_{2} / J_{1} \lesssim 0.6$, and thence to a collinear striped phase for $J_{2} / J_{1} \gtrsim 0.6$. The synthesis of layered magnetic materials that might be described by the square-lattice $J_{1}-J_{2}$ model $^{8,9}$ has also encouraged further theoretical studies of this model. Although the square-lattice spin- $\frac{1}{2} J_{1}-J_{2}$ HAFM has been intensively studied for over 20 years, no consensus yet exists on such fundamental issues as the nature of the QP phase and of the type of transition into it. In particular, there is still controversy over whether the scenario of deconfined criticality holds for this model (see, e.g., Refs. 4 and 5).

Related magnetic systems may shed light on this controversy and one related model that has received attention recently has been the HAFM on the honeycomb lattice. One reason for this interest is that a spin-liquid phase has been found for the exactly solvable Kitaev model, ${ }^{10}$ in which the spin- $\frac{1}{2}$ particles reside on a honeycomb lattice. The honeycomb lattice is also relevant to the active research field of graphene, where Hubbard-like models on this lattice may be appropriate to describe the relevant physics. ${ }^{11}$ Interestingly, Meng et al. ${ }^{12}$ found clear evidence that the quantum fluctuations are strong enough to establish an insulating spin-liquid phase between the nonmagnetic metallic phase and the AFM Mott insulator for the Hubbard model on the honeycomb lattice at moderate values of the Coulomb repulsion $U$. At very large values of $U$, the latter phase corresponds to the HAFM on the bipartite honeycomb lattice that contains a ground state showing Néel long-range order (LRO). However, higher-order terms in the $t / U$ expansion of the Hubbard model may lead to frustrating exchange couplings in the corresponding spin-lattice model where the HAFM with nn exchange is the leading term in the large- $U$ expansion. This unexpected result, ${ }^{12}$ and other similar work, ${ }^{13}$ have stimulated interest in the frustrated HAFM on the honeycomb lattice (see, e.g., Refs. 14-17). Indeed, the available literature suggests a frustration-induced QP phase for the frustrated spin- $\frac{1}{2}$ HAFM on the honeycomb lattice. ${ }^{14-19}$ These theoretical findings are consistent with the recent experimental observations that the spin- $\frac{3}{2}$ honeycomb-lattice $\mathrm{HAFM} \mathrm{Bi} 3 \mathrm{Mn}_{4} \mathrm{O}_{12}\left(\mathrm{NO}_{3}\right)$ shows a spin-liquid-like behavior at temperatures much lower than the Curie-Weiss temperature. ${ }^{20}$

One approach to shed additional light on the QP regions of such $J_{1}-J_{2}$ models is to extend its parameter space by the inclusion of next-next-nearest-neighbor (nnnn) couplings $J_{3}$ as well. This yields the $J_{1}-J_{2}-J_{3}$ model (see, e.g., Ref. 21 and references cited therein)

$$
H=J_{1} \sum_{\langle i, j\rangle} \mathbf{s}_{i} \cdot \mathbf{s}_{j}+J_{2} \sum_{\langle\langle i, k\rangle\rangle} \mathbf{s}_{i} \cdot \mathbf{s}_{k}+J_{3} \sum_{\langle\langle\langle i, l\rangle\rangle\rangle} \mathbf{s}_{i} \cdot \mathbf{s}_{k},
$$

where $i$ runs over all lattice sites, and $j$ runs over all nn sites, $k$ over all nnn sites, and $l$ over all nnnn sites to $i$, respectively, counting each bond once and once only. Each site $i$ of the lattice carries a spin- $\frac{1}{2}$ particle with spin operator $\mathbf{s}_{i}$. Here we study the spin- $\frac{1}{2} J_{1}-J_{2}-J_{3}$ HAFM model of Eq. (1) on the honeycomb lattice. ${ }^{14-19}$ The lattice and the exchange bonds are illustrated in Fig. 1 (right panel), where the quasiclassical Néel and collinear striped phases are also shown. Classically, this system exhibits the two collinear phases illustrated in Fig. 1 (right panel) as well as a spiral phase. These phases meet in a triple point at $J_{2}=J_{3}=J_{1} / 2$ (see, e.g., Refs. 14 and 19). Henceforth we set $J_{1} \equiv 1$ and restrict ourselves to the case $J_{2}=J_{3} \equiv \kappa J_{1}$. The motivation to focus on $J_{2}=J_{3}$ also comes from the classical case, where the honeycomblattice model and the square-lattice $J_{1}-J_{2}$ HAFM model have 

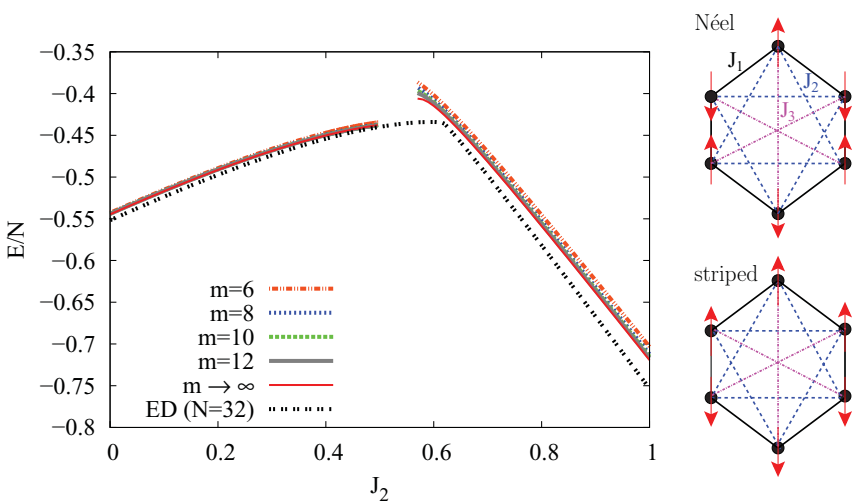

FIG. 1. (Color online) Left: Results for the gs energy, $E / N\left(J_{1} \equiv\right.$ 1 and $\left.J_{3}=J_{2}\right)$ via ED $(N=32), \operatorname{CCM} \operatorname{LSUB} m(m=6,8,10,12)$, and extrapolated $m \rightarrow \infty$ (see text). Right: The $J_{1}-J_{2}-J_{3}$ honeycomb model for the Néel and the collinear striped model states. The arrows represent spins located on lattice sites $\bullet$.

similar ground-state (gs) properties, namely: (i) there is a direct transition between the Néel and the collinear striped states at $\kappa=0.5$; and (ii) the classical ground state is highly degenerate at the transition point. Hence, the two systems are very similar classically, and so the honeycomb system is a likely candidate to exhibit a QP phase and possibly also a deconfined critical point between this QP phase and the quasiclassical phases. Moreover, we note again that non-zero $J_{3}$ bonds are likely from higher-order terms in the $t / U$ expansion of the Hubbard model at moderate $U$ values. ${ }^{12}$

The treatment of highly frustrated quantum magnets is notoriously challenging since only a relatively few accurate methods exist. Recent approaches such as those based on projected entangled pair states ${ }^{22}$ are not yet accurate enough. The finite-size extrapolation of numerical exact data for finite-lattice systems, used successfully for the square-lattice model, ${ }^{2,7}$ is also less efficient for the honeycomb lattice since (i) the unit cell contains two sites, (ii) nnnn couplings $J_{3}$ are included, and (iii) there are only a few finite lattices with full point group symmetry. ${ }^{19}$ Naturally, quantum Monte Carlo methods are severely limited in the presence of frustration due to the infamous "sign problem." The coupled-cluster method (CCM) when evaluated to high orders of approximation provides a reasonably accurate approach to determine the position of QPT points, ${ }^{5,21,23-26}$ as well as providing evidence on the nature of such QP phases. ${ }^{5}$ Here we use this method together with complementary results provided by the exact diagonalization (ED) technique for a finite lattice of $N=32$ sites.

The CCM is a size-extensive method that provides results in the limit $N \rightarrow \infty$ from the outset. The CCM requires us to input a model (or reference) state, ${ }^{27-29}$ with respect to which the quantum correlations are expressed. Here we use the Néel and striped model states shown in Fig. 1 (right panel). We use also the well-tested localized lattice-animal-based subsystem $(\mathrm{LSUB} m)$ truncation scheme in which all multispin correlations are retained in the CCM correlation operators over all distinct locales on the lattice defined by $m$ or fewer contiguous sites. The method of solving for higher orders of LSUBm approximations is discussed in detail in Refs. 27 and 28. The number of independent spin configurations taken into account in the correlation operator increases rapidly with the truncation index $m$. For example, at the highest level of approximation used here, namely LSUB12, we take into account 750490 such configurations. To obtain results in the exact $m \rightarrow \infty$ limit the raw LSUB $m$ data must be extrapolated. Although there are no exact extrapolation rules, a great deal of experience has been garnered by now for the gs energy and for the magnetic order parameter (sublattice magnetization) $M$. For the gs energy per spin a well-tested and very accurate extrapolation ansatz (see, e.g., Refs. 5,6,23-26,28,30) is $E(m) / N=a_{0}+a_{1} m^{-2}+$ $a_{2} m^{-4}$, while for the magnetic order parameter $M$ different schemes have been employed for different situations. ${ }^{31}$ Here we use $M(m)=b_{0}+b_{1} m^{-1 / 2}+b_{2} m^{-3 / 2}$. This scheme has been found to be appropriate for systems showing a gs order-disorder QPT (see, e.g., Refs. 5,21,25,26). Since the hexagon is an important structural element of the honeycomb lattice we use for the extrapolations only $\mathrm{LSUB} m$ data with $m \geqslant 6$.

CCM results for the gs energy per spin $E / N$ are shown in Fig. 1 using both the Néel and collinear striped model states. They are clearly well-converged for all values of $J_{2}$ shown. The corresponding extrapolated LSUB $\infty$ results are also shown in the regimes where real solutions exist for the entire data set, for each choice of reference state. These results compare well to those from ED calculations for $N=32$, also shown in Fig. 1. As is usually the case the $\mathrm{CCM} \mathrm{LSUB} m$ results for finite $m$ values for a given phase extend beyond the actual $\operatorname{LSUB} \infty$ transition point. If the $\mathrm{CCM}$ results in Fig. 1 are terminated at the $\mathrm{LSUB} \infty$ critical points obtained from Fig. 2 as discussed below, the agreement between the CCM and ED gs energy results is even more striking (and recall too that the ED results have undergone no finite-size scaling to the infinite-lattice limit). The CCM results show a clear intermediate region around $\kappa=0.5$ in which neither of the quasiclassical AFM states is stable. The ED results demonstrate a "kink-like" behavior in $E / N$ at $\kappa \approx 0.6$, (cf. Refs. 7 and 14), which is a first hint of a first-order QPT (and see the discussion below).

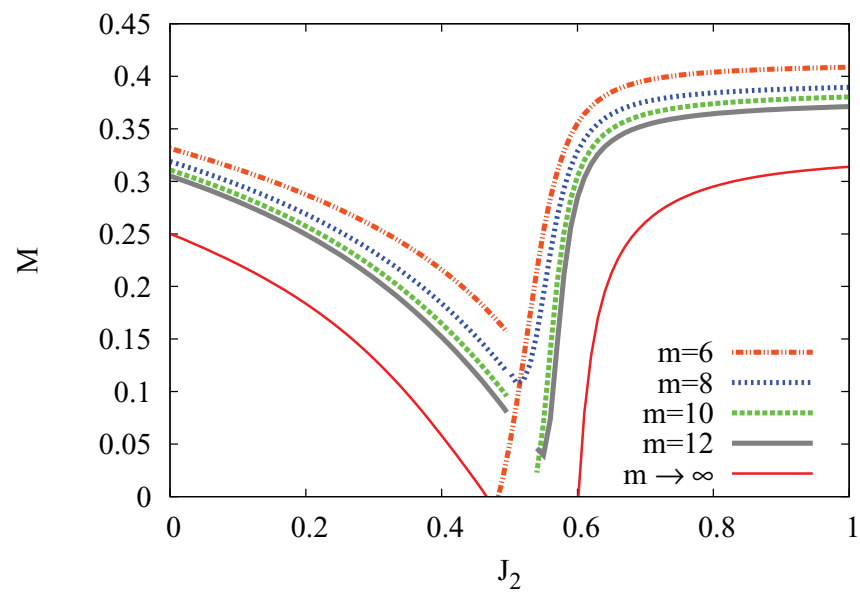

FIG. 2. (Color online) CCM results for the gs order parameter, $M$ ( $J_{1} \equiv 1$ and $J_{3}=J_{2}$ ) for the Néel and striped phases using LSUB $m$ ( $m=6,8,10,12)$, and extrapolated $m \rightarrow \infty$ (see text). 


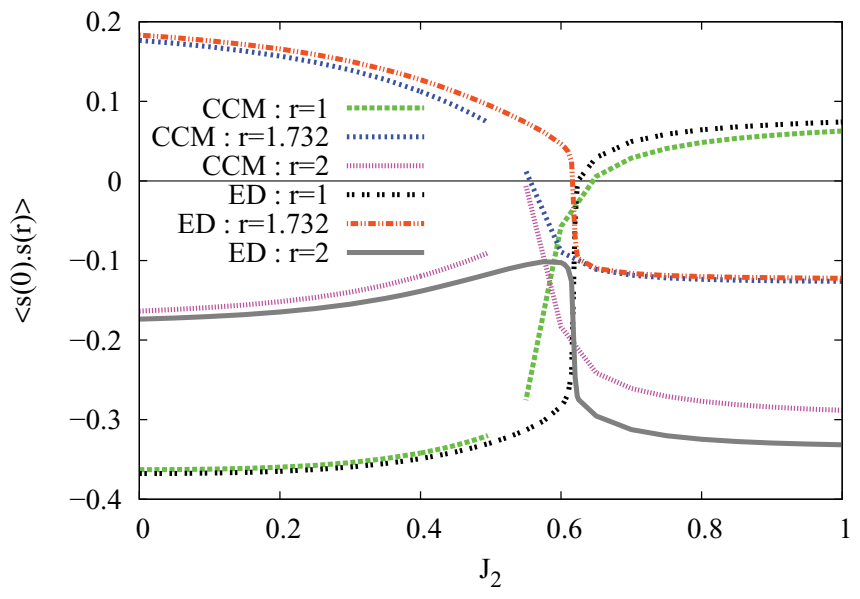

FIG. 3. (Color online) CCM LSUB 12 and $N=32$ ED results for the spin-spin correlation function $\langle\mathbf{s}(0) \cdot \mathbf{s}(r)\rangle\left(J_{1} \equiv 1\right.$ and $\left.J_{3}=J_{2}\right)$. Values $r=1,1.732,2$ correspond, respectively, to $\mathrm{nn}, \mathrm{nnn}$, and nnnn pairs of spins.

We now discuss the magnetic order parameter $M$ to investigate the stability of quasiclassical magnetic LRO. CCM results for $M$ are shown in Fig. 2. The extrapolated Néel order parameter vanishes at $\kappa_{c_{1}} \approx 0.466$, whereas its collinear striped counterpart goes to zero at $\kappa_{c_{2}} \approx 0.601$. These values can be considered as CCM estimates for the QCPs of the model. ${ }^{32}$ They are in good agreement with those recently obtained by the Schwinger-boson approach ${ }^{14}$, the functional renormalization group approach, ${ }^{17}$ and by an ED approach. ${ }^{16}$ From the shape of the order-parameter curve in Fig. 2 it seems probable that the transition at $\kappa_{c_{1}}$ is continuous, whereas the very steep fall near $\kappa_{c_{2}}$ may indicate a first-order transition. This is supported by the spin-spin correlations functions shown in Fig. 3, which also show a similar "smooth" versus "steep" behavior near the points $\kappa_{c_{1}}$ and $\kappa_{c_{2}}$, respectively. Moreover, the CCM and ED data presented in Fig. 3 are in good quantitative agreement, which supports the conclusions that have been drawn above regarding the likely nature of the QPTs.

Hitherto we have gathered strong evidence about the region of the intermediate nonmagnetic quantum phase by using the high-order CCM and ED techniques. However, the question remains as to what is the nature of this phase. We note that numerical evidence was found very recently of a plaquette valence-bond crystal (PVBC) phase near $\kappa=0.5$ (Ref. 16). Note that such a PVBC phase is a strong candidate for the QP phase of the $J_{1}-J_{2}$ model on the square lattice. ${ }^{3-5}$ To analyze the possibility of such a PBVC phase we first consider a generalized susceptibility $\chi_{F}$ that describes the response of the system to a "field" operator $F$ (see, e.g., Refs. 4 and 5). We thus add a field term $F=\delta \hat{O}$ to the Hamiltonian (1), where $\hat{O}$ is an operator that in our case corresponds to the possible PVBC order illustrated in Fig. 4 (right panel), and which hence breaks the translational symmetry of $H$. We now calculate the energy per site $E(\delta) / N=e(\delta)$ for the perturbed Hamiltonian $H+F$, by using the CCM for both the Néel and the collinear striped reference states, and define the susceptibility as $\chi_{F} \equiv-\left.\left[\partial^{2} e(\delta) / \partial \delta^{2}\right]\right|_{\delta=0}$. Rather less empirical experience is available regarding the extrapolation

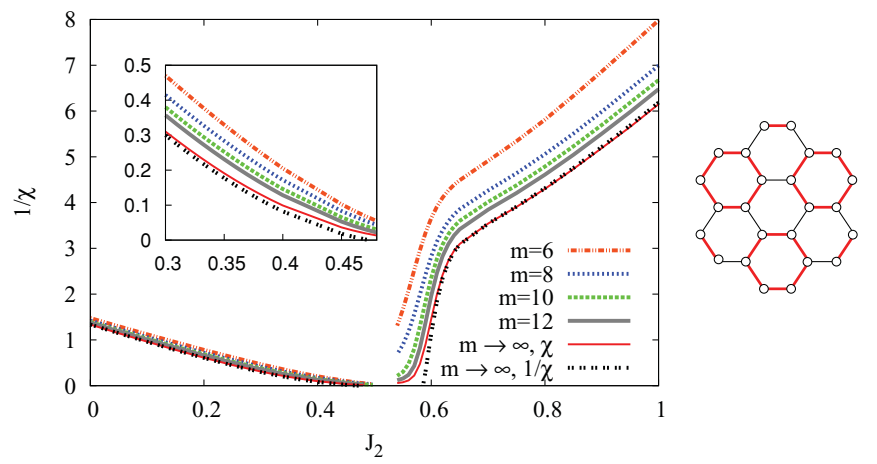

FIG. 4. (Color online) Left: Results for the inverse plaquette susceptibility, $1 / \chi\left(J_{1} \equiv 1\right.$ and $\left.J_{3}=J_{2}\right)$ via CCM LSUBm $(m=$ $6,8,10,12$ ), and extrapolated $(m \rightarrow \infty)$ for both $\chi$ and $1 / \chi$ (see text). Right: The perturbations (fields) $F=\delta \hat{O}$ for the plaquette susceptibility $\chi$. Thick (red) and thin (black) lines correspond, respectively, to strengthened and weakened nn exchange couplings, where $\hat{O}=\sum_{\langle i, j\rangle} a_{i j} \mathbf{s}_{i} \cdot \mathbf{s}_{j}$, and the sum runs over all nn bonds, with $a_{i j}=+1$ and -1 for thick (red) and thin (black) lines, respectively.

of the CCM LSUB $m$ data for $\chi_{F}$ than for other quantities such as the gs energy or $M$. However, we have tested several extrapolation schemes and found that the extrapolation used for the gs energy [i.e., $\chi_{F}(m)=c_{0}+c_{1} m^{-2}+c_{2} m^{-4}$ ] fits the data most accurately. For a crosscheck of this extrapolation scheme we also use corresponding extrapolation of the inverse susceptibility [i.e., $\chi_{F}^{-1}(m)=d_{0}+d_{1} m^{-2}+d_{2} m^{-4}$ ] and find consistent results as shown in Fig. 4. An instability of the gs against the perturbation $F$ is indicated by a divergence of $\chi_{F}$ or equivalently a zero point of $\chi_{F}^{-1}$. This fact might favor slightly the extrapolation of $\chi_{F}^{-1}$ when searching for such instabilities. Our results are in accordance with those of Ref. 16 and they clearly favor a PVBC intermediate quantum phase instead of a structureless spin-liquid phase. The extrapolated inverse susceptibility vanishes on the Néel side at $\kappa \approx 0.473$ and on the collinear striped side at $\kappa \approx 0.586$. These values are in very good agreement with the critical values $\kappa_{c_{1}} \approx 0.466$ and $\kappa_{c_{2}} \approx 0.601$ already found by the CCM order parameter. Thus, it is most likely that the PVBC phase occurs at (or is very close to) the transition points where the quasiclassical magnetic LRO breaks down. Although there is a steep downturn of $\chi_{F}^{-1}$ at $\kappa_{c_{2}}$, we find a smooth behavior for $\chi_{F}^{-1}$ at $\kappa_{c_{1}}$. This evidence strongly supports our conclusion drawn from Figs. 1, 2, and 3 that the phase transition is of first-order type at $\kappa_{c_{2}}$ while that at $\kappa_{c_{1}}$ is of continuous type. We should point out, however, that there exist generic arguments that the phase transitions in models with $S U(2)$-symmetric deconfined critical points should be first order. ${ }^{33}$ Nevertheless, these arguments are based on effective field theories, while our own calculations are based on the actual lattice model itself. While we can never entirely exclude the possibility of a sufficiently weak first-order transition rather than a continuous one, we find no evidence that the transition at $\kappa_{c_{1}}$ is first order, although that at $\kappa_{c_{2}}$ clearly is.

The possibility of deconfined quantum criticality for the frustrated honeycomb HAFM was pointed out in Ref. 1. Supporting evidence for the $J_{1}-J_{2}-J_{3}$ model on the honeycomb lattice has been found recently in Ref. 16. Our CCM 
results are seen to be highly converged and to agree well with those of the ED method. Our results for the gs energy, magnetic order parameter, spin-spin correlation function, and plaquette susceptibility point to collinear phases separated by a magnetically disordered phase for $0.47 \lesssim \kappa \lesssim 0.60$. We have shown that this intermediate phase is likely to be of plaquette valence-bond crystalline (PVBC) type. Although we find indications of a first-order transition at $\kappa_{c_{2}} \approx 0.60$, the transition between the Néel and the PVBC phases at $\kappa_{c_{1}}$ appears continuous. Since the Néel and the PVBC phases break different symmetries and we have shown that they are likely to meet at $\kappa_{c_{1}} \approx 0.47$, our results present strong evidence that the frustrated spin- $\frac{1}{2}$ HAFM on the honeycomb lattice contains a continuous QPT described by the scenario of deconfined quantum criticality.

We thank the University of Minnesota Supercomputing Institute for the grant of supercomputing facilities. J.R. thanks A. Läuchli for fruitful discussions. One of the authors (D.J.J.F.) acknowledges and thanks the European Science Foundation (ESF) for financial support via the research network program entitled "Highly Frustrated Magnetism".
${ }^{1}$ T. Senthil, A. Vishwanath, L. Balents, S. Sachdev, and M. P. A. Fisher, Science 303, 1490 (2004); T. Senthil, L. Balents, S. Sachdev, A. Vishwanath, and M. P. A. Fisher, Phys. Rev. B 70, 144407 (2004).

${ }^{2}$ H. J. Schulz, T. A. L. Ziman, and D. Poilblanc, J. Phys. I 6, 675 (1996).

${ }^{3}$ M. E. Zhitomirsky and K. Ueda, Phys. Rev. B 54, 9007 (1996).

${ }^{4}$ J. Sirker, Zheng Weihong, O. P. Sushkov, and J. Oitmaa, Phys. Rev. B 73, 184420 (2006).

${ }^{5}$ R. Darradi, O. Derzhko, R. Zinke, J. Schulenburg, S. E. Krüger, and J. Richter, Phys. Rev. B 78, 214415 (2008).

${ }^{6}$ J. Reuther and P. Wölfle, Phys. Rev. B 81, 144410 (2010).

${ }^{7}$ J. Richter and J. Schulenberg, Eur. Phys. J. B 73, 117 (2010).

${ }^{8}$ R. Melzi, P. Carretta, A. Lascialfari, M. Mambrini, M. Troyer, P. Millet, and F. Mila, Phys. Rev. Lett. 85, 1318 (2000).

${ }^{9}$ H. Rosner, R. R. P. Singh, W. H. Zheng, J. Oitmaa, S.-L. Drechsler, and W. E. Pickett, Phys. Rev. Lett. 88, 186405 (2002).

${ }^{10}$ A. Kitaev, Ann. Phys. (NY) 321, 2 (2006); G. Baskaran, S. Mandal, and R. Shankar, Phys. Rev. Lett. 98, 247201 (2007); J. Chaloupka, G. Jackeli, and G. Khaliullin, ibid. 105, 027204 (2010).

${ }^{11}$ A. H. Castro Neto, F. Guinea, N. M. R. Peres, K. S. Novoselov, and A. K. Geim, Rev. Mod. Phys. 81, 109 (2009).

${ }^{12}$ Z. Y. Meng, T. C. Lang, S. Wessel, F. F. Assaad, and A. Muramatsu, Nature (London) 464, 847 (2010).

${ }^{13}$ H. Y. Yang and K. P. Schmidt, Europhys. Lett. 94, 17004 (2011).

${ }^{14}$ D. C. Cabra, C. A. Lamas, and H. D. Rosales, Phys. Rev. B 83, 094506 (2011).

${ }^{15}$ H. Mosadeq, F. Shahbazi, and S. A. Jafari, J. Phys. Condens. Matter 23, 226006 (2011).

${ }^{16}$ A. F. Albuquerque, D. Schwandt, B. Hetényi, S. Capponi, M. Mambrini, and A. M. Läuchli, e-print arXiv:1102.5325.

${ }^{17}$ J. Reuther, D. A. Abanin, and R. Thomale, e-print arXiv:1103.0859.

${ }^{18}$ A. Mattsson, P. Fröjdh, and T. Einarsson, Phys. Rev. B 49, 3997 (1994).
${ }^{19}$ J. B. Fouet, P. Sindzingre, and C. Lhuillier, Eur. Phys. J. B 20, 241 (2001).

${ }^{20}$ S. Okubo, F. Elmasry, W. Zhang, M. Fujisawa, T. Sakurai, H. Ohta, M. Azuma, O. A. Sumirnova, and N. Kumada, J. Phys.: Conf. Ser. 200, 022042 (2010).

${ }^{21}$ J. Reuther, P. Wölfle, R. Darradi, W. Brenig, M. Arlego, and J. Richter, Phys. Rev. B 83, 064416 (2011).

${ }^{22}$ V. Murg, F. Verstraete, and J. I. Cirac, Phys. Rev. B 79, 195119 (2009).

${ }^{23}$ R. Darradi, J. Richter, and D. J. J. Farnell, Phys. Rev. B 72, 104425 (2005).

${ }^{24}$ D. Schmalfuß, R. Darradi, J. Richter, J. Schulenburg, and D. Ihle, Phys. Rev. Lett. 97, 157201 (2006).

${ }^{25}$ R. F. Bishop, P. H. Y. Li, R. Darradi, J. Schulenburg, and J. Richter, Phys. Rev. B 78, 054412 (2008).

${ }^{26}$ J. Richter, R. Darradi, J. Schulenburg, D. J. J. Farnell, and H. Rosner, Phys. Rev. B 81, 174429 (2010).

${ }^{27}$ C. Zeng, D. J. J. Farnell, and R. F. Bishop, J. Stat. Phys. 90, 327 (1998).

${ }^{28}$ R. F. Bishop, D. J. J. Farnell, S. E. Krüger, J. B. Parkinson, J. Richter, and C. Zeng, J. Phys. Condens. Matter 12, 6887 (2000).

${ }^{29}$ R. F. Bishop, D. J. J. Farnell, and J. B. Parkinson, Phys. Rev. B 58, 6394 (1998).

${ }^{30}$ R. F. Bishop, P. H. Y. Li, R. Darradi, and J. Richter, J. Phys. Condens. Matter 20, 255251 (2008).

${ }^{31}$ Note that for unfrustrated models the extrapolation $M(m)=$ $b_{0}+b_{1} m^{-1}+b_{2} m^{-2}$ is more appropriate (Ref. 28). This scheme gives the best result, $M \approx 0.273$, for the pure nn HAFM on the honeycomb lattice $\left(J_{2}=J_{3}=0, J_{1}=1\right)$.

${ }^{32}$ To estimate the accuracy within the used extrapolation scheme we have also determined $\kappa_{c_{1}}$ and $\kappa_{c_{2}}$ using LSUB $m$ with $m=\{6,8,10\}$, which gives $\kappa_{c_{1}} \approx 0.448$ and $\kappa_{c_{2}} \approx 0.601$. The values $\kappa_{c_{1}}$ and $\kappa_{c_{2}}$ of course depend also on the extrapolation scheme employed.

${ }^{33}$ A. B. Kuklov, M. Matsumoto, N. V. Prokof'ev, B. V. Svistunov, and M. Troyer, Phys. Rev. Lett. 101, 050405 (2008). 